American Journal of Infectious Diseases 2 (4): 193-196, 2006

ISSN 1553-6203

(C) 2006 Science Publications

\title{
The Effect of Zinc and "Health Belief Model" Based Education on Common Cold Prevention in Soldiers
}

\author{
${ }^{1}$ Arash Pooya, ${ }^{2}$ Ahmad Mahmoudian, ${ }^{3}$ Mohamad Mahdi Hazavei, ${ }^{2}$ Ziba Farajzadegan and \\ ${ }^{2}$ Mohammad Arash Ramezani \\ ${ }^{1}$ Department of Community Medicine, School of Medicine,Hamedan University of Medical Sciences \\ Shahid Fahmideh Boulevard, Hamedan, Iran \\ ${ }^{2}$ Department of Community Medicine, School of Medicine, Isfahan University of Medical Sciences \\ ${ }^{3}$ Department of Health Education, School of Health, Isfahan University of Medical Sciences
}

\begin{abstract}
The purpose of this study was to determine the effect of "Zinc" \& "Health Belief model" based education on viral upper respiratory tract infections in soldiers in Isfahan - 2004 - 2005. It was a double - blinded randomized controlled trial, which was done among a group of soldiers by census. Soldiers were randomly divided into four groups. On the other hand four preventive measures were assumed regarding presence of education and zinc prescription, which were randomly allocated to four groups. Subsequently education was performed to change the health behavior of soldiers. Meanwhile tablets were distributed among all groups of soldiers. Data regarding incidence and duration of symptoms were gathered by different questionnaires filled by soldiers themselves and doctors and finally analyzed by SPSS 11.5 software through $\chi^{2}$, t-test and logistic regression. Common cold incidence in groups receiving zinc vs. placebo was significantly less. $(\mathrm{P}=0.001)$. Groups, which received education comparing to those, which didn't, were statistically the same 'regarding the incidence of common cold $(\mathrm{P}=0.37)$. Mean duration of symptoms was less in groups receiving zinc comparing to the groups, which received placebo as it was similar regarding education $(\mathrm{P}=0.007$, 0.01 ). All of all 'this study showed that zinc consumption can decrease the incidence and duration of common cold. Education could promote some preventive behaviors and compliance to tablet consumption but had no effect on incidence. Decreased incidence by zinc consumption could be in part related to education through increased compliance for tablet consumption.
\end{abstract}

Key words: Common cold, zinc, health education, incidence, prevention

\section{INTRODUCTION}

Common cold is the most common upper respiratory tract disease and a self -limited on ${ }^{[1]}$ which accounts for 21 million lost days ( for work) and 25 million lost days (for education) in the United States ,this way being the most common reason for absence from school or work place ${ }^{[2]}$. This problem is especially prominent when there is a large group of people living together and sometimes having close contacts like pilgrims to Haj and soldiers. Many measures including vitamin $\mathrm{C}$, changing health behavior and zinc usage have been used to prevent this disease.

Once that is controversy about it, is Zinc. In 2004 Walker showed that Zinc could decrease the incidence rate of diarrhea, pneumonia and possibly malaria ${ }^{[3]}$. In another study performed in 2001 by Turner, intranasal spray of Zinc Gluconate was of no benefit in preventing common cold, which was induced by rhinovirus inoculation $^{[4]}$. Sazawal study 1998 showed that Zinc supplementation could decrease the incidence of common cold among preschool children ${ }^{[5]}$. Zinc can inhibit viral capsid proteins formation and as a result inhibits In-vitro multiplication of different viruses including Herpes simplex virus, Foot and mouth disease virus, Polio virus and Rhinovirus.

Other mechanisms by which zinc affects common cold are viral attachment inhibition, cell membrane fixation, inducing $\gamma$-interferon production and prostaglandin metabolism inhibition ${ }^{[6]}$.

Meanwhile "Health belief model" could be used to describe behavior and respectively to change health behaviors and prevent common cold. In this health education model each person is affected by perceived

Corresponding Address: $\quad$ Department of Community Medicine, School of Medicine,Hamedan University of Medical Sciences, Shahid Fahmideh Boulevard, Hamedan,Iran, Tel: +988114230934, +989133008663 
threats, perceived benefits and barriers and cues to action $^{[7,8]}$. Developing a hand-washing program by Ryan for soldiers in 2001 resulted in decreased viral infections ${ }^{[9]}$. In this study we tried to assess the effectiveness of Zinc prescription and Health belief model based education on preventing common cold and decreasing the severity of the disease in soldiers.

\section{MATERIALS AND METHODS}

In this study, which was, a double blind randomized controlled trial a group of soldiers in a garrison in Isfahan were enrolled by census in 2005 spring.

Inclusion criterion was acceptance of the studied individual for Zinc- sulfate usage. Exclusion criteria were no tendency for remaining in the study and intolerable gastrointestinal complications.

Practitioners and commanders of the garrison were informed about the project including allocation of interventions and data gathering, in different sessions. All soldiers (225 individuals) were enrolled as sample. These soldiers lived in two independent 24-hour sanatoriums. Residents of each sanatorium were randomly divided into two groups. Each soldier performed this randomization through picking up an even or odd number. This way we had four groups to which options mentioned below were allocated:

* Health belief model based education plus Zinc sulfate prescription two times a day for three weeks.

* Health belief model based education plus placebo prescription two times a day for three weeks.

* Only Zinc sulfate prescription two times a day for three weeks.

* Only placebo prescription two times a day for three weeks.

Options 1 and 2 were allocated randomly to one sanatorium and options 3 and 4 to the other one. As we could not educate just half of a group, association of options 1 and 2 and therefore 3 and 4 were inevitable.

A session was managed by "lecturing" and "question and answer" methods regarding educational behavioral objectives, for groups 1 and 2 .

These educational behavioral objectives were developed according to "health belief model " aspects. In addition, a pamphlet and a poster were used in educating these groups. All groups were informed about the usage of Zinc sulfate tablets and received drug packages.

Two days after performing the education and distribution of tablets, surveillance for common cold was started by the garrison practitioners and continued for six weeks. After a six-week surveillance, soldiers regarding practiced health behavior and drug usage answered a questionnaire.

Meanwhile drug packages were collected and unused tablets were counted. Finally the incidence rate of viral upper respiratory tract infections during the surveillance period was calculated in different groups and compared with each other.

Gathered data was analyzed by SPSS v.11.5 through logistic regression, independent samples t-test, odds ratio analysis and $\chi^{2}$. Meanwhile, best case-worst case analysis was done to analyze the effect of missed data.

\section{RESULTS}

Relative frequency of common cold was highest in group $4(88.9 \%)$ and less in groups 2,3 and 1 respectively( Table 1).

To assess the effectiveness of Zinc in preventing common cold, groups of Zinc consumption $(1,3)$ were compared with groups of placebo consumption $(2,4)$. Results showed (table 2) that zinc was effective. Relative risk $=0.72$, 95\%Confidence interval $=0.343$ 0.823 Also to asses the effectiveness of HBM based education in preventing common cold, educated groups $(1,2)$ were compared with groups of no education $(3,4)$. Table 3 illustrated no effectiveness for HBM based education. Relative risk $=0.91 \quad 0.95 \%$ Confidence interval 0.493-1.313

In addition, best case -worst case analysis was carried out to solve the problem of missing data. By this analysis, results were the same as what is mentioned above.

Mean duration of common cold was significantly different between zinc group and placebo group: ( $P$ value $=0.007$ ) as it was between group of education and group of no education ( $\mathrm{P}$ value $=0.008$ ).

On the other hand mean number of consumed pills was significantly more in-group of education (comparing to the group of no education): $\mathrm{P}$ value $=0.01$.

Although two preventive behaviors (avoiding hand shaking and handkerchief usage in common cold) were found more frequent among group of education comparing to the group of no education ( $P$ value $=0.02,0.002$ respectively), non of the preventing behaviors including hand washing, avoiding hand shaking, handkerchief and mask usage in addition to relaxation, hydration and exercise were related to common cold incidence rate $(\mathrm{P}$ value $>0.05$ for all variables). 
Am. J. Infect. Dis., 2 (4): 193-196, 2006

Table 1: Relative frequency of common cold in studied groups

\begin{tabular}{llllll}
\hline & Group number & & & Total \\
& $1($ Zn+education) & 2(Placebo+ Education) & $3(\mathrm{Zn})$ & 4(Placebo) & \\
\hline Absence of common cold & $25(42.4 \%)$ & $13(21.7 \%)$ & $12(37.5 \%)$ & $3(11.1 \%)$ & $53(29.8 \%)$ \\
Presence of common cold & $34(57.6 \%)$ & $47(78.3 \%)$ & $20(62.5 \%)$ & $24(88.9 \%)$ & $125(70.2 \%)$ \\
Total & $59(100 \%)$ & $60(100 \%)$ & $32(100 \%)$ & $27(100 \%)$ & $178(100 \%)$ \\
\hline P & & &
\end{tabular}

$\mathrm{P}$ value $=0.015$

Table 2: Frequency of common cold among zinc consumers and placebo consumers

\begin{tabular}{llll}
\hline & Group & & Total \\
& Zinc & Placebo & \\
\hline Absence of common cold & 37 & 16 & 53 \\
Presence of common cold & 54 & 71 & 125 \\
Total & 91 & 87 & 178 \\
\hline
\end{tabular}

$\mathrm{P}$ value $=0.001$

Relative risk $=0.72,95 \%$ Confidence interval $=0.343-0.823$

Table 3: Frequency of common cold among groups of education and groups of no education

\begin{tabular}{llll}
\hline & Group & Total \\
& Education & No education & \\
\hline Absence of common cold & 38 & 15 & 53 \\
Presence of common cold & 81 & 44 & 125 \\
Total & 119 & 59 & 178 \\
\hline
\end{tabular}

Pvalue $=0.371$

Relative risk $=0.91$ 0.95\%: Confidence interval 0.493-1.313

\section{DISCUSSION}

The aim of this study was to assess the effectiveness of zinc sulfate consumption and health belief model based education on preventing viral upper respiratory tract infections among soldiers. In this study, zinc sulfate consumption appeared to be effective in preventing common cold while education had indirect effects.

In 1998 Sazawal study showed that Zinc supplementation could decrease the incidence of common cold among preschool children ${ }^{[5]}$. A study performed in 2002 by Mc Elroy showed that zinc gluconate lozenge could be effective in decreasing the incidence and duration of common cold ${ }^{[10]}$. In another study performed in 2001 by Turner, intranasal spray of Zinc Gluconate was of no benefit in preventing common cold which was induced by rhinovirus inoculation $^{[4]}$.

Jackson performed a meta-analysis using 10 clinical trials and concluded that zinc gluconate lozenges was of no benefit treating common $\operatorname{cold}^{[11]}$. Below, some reasons of this controversy in different researches are listed:

Level of zinc deficiency is different in different areas thus affecting the results of these studies in different ways. In addition, the viral etiology of common cold differs in different areas and seasons and results some controversies. Also, the dose and form of the drugs applied in these studies were different. All together, it seems that oral prescription of zinc shows superior effects comparing topical usage of this drug, regarding prevention of common cold.

In our study, statistical analysis showed that HBM based education had no direct effect on decreasing common cold incidence. While group of education had used more pills (more compliance), it could be concluded that a part of relationship between zinc and common cold incidence is due to education (indirectly).

Comparing health behaviors in-group of education and group of no education, just two behaviors (handkerchief usage and no hand -shaking) were more frequent in the first group while non of the health behaviors were related to common cold incidence. Developing a hand washing program by Ryan for soldiers in 2001 resulted in decreased viral infections ${ }^{[9]}$. In 1993 Sattar et al showed that hand washing had a definite role in preventing rhinovirus transmission ${ }^{[12]}$.

In a study performed by Niffenegger in 1997, hand washing resulted in decreased incidence of infectious diseases in children's care centers ${ }^{[13]}$.

Performing executive programs versus an educational program was the difference between our study and the above mentioned studies. This way finding no relationship between education and common cold incidence rate could be attributed to no or mild behavioral changes in our study. In this study, zinc consumption was associated with decreased duration of common cold while Belongia et al in 2000 found no relationship between low-dose intranasal zinc sulfate spray usage and duration of common cold ${ }^{[14]}$. This difference could be justified regarding the form of the drug and may be the dose applied.

Group of education used more pills comparing to the group of no education, this could mean more compliance among the first group.

All together it seems that oral usage of zinc could decrease both incidence and duration of common cold. HBM based education could promote two health behaviors (handkerchief usage and no hand-shaking) but could not decrease the incidence of common cold 
through this process. On the other hand, education resulted in increased compliance and thus decreased common cold incidence. Respecting what we have achieved, it seems that combining educational and pharmacological methods results in additive effects and increases the effectiveness of each method. Results of this study could be applied in dense groups like pilgrims to Haj, soldiers and dormitory residents.

\section{ACKNOWLEDGEMENT}

We would like to thank medical university of Isfahan for supporting the study. Also special thanks to Dr. M.Dehghannejhad and Vatanpour garrison commanders and soldiers who really helped us during execution. In addition we appreciate Dr. R.Rouzbehani for his helpful comments during proposal writing and execution and Dr.Varshosaz for preparing zinc sulfate and placebo tablets. Finally we greatly appreciate Mrs. L. Gheiratmand for her nice assistance during all stages of the study.

\section{REFERENCES}

1. Hendley, J.O., 2000. The Common Cold. Goldman Lee, Bennett J. Cecil, Text Book of Medicine: WB Saunders Company, pp: 1790-92.

2. Turner, R.B., 2004. The Common Cold. Behrman R, Kliegman. Nelson Text Book of Pediatrics. Philadelphia,W.B Saunders Company, pp:1389-91.

3. Walker, C.F. and R.E. Black, 2004. Zinc and the risk for infectious disease. Annu Rev. Nutr., 24: 255-75.

4. Turner, R.B., 2001. Ineffectiveness of internal zinc gluconate for prevention of Clin. Infect. Dis., 33: 1865-1870.
5. Sazawal, S. and R.E. Black, 1998. Zinc supplementation reduces the incidence of acute lower respiratory infections. Sci. Direc., 102: 1-15.

6. Sherif, B., 1997. Zinc and the common cold: Are we close to a cure? Nutr. and Epidemiol., 13: 708709.

7. Shojaizadeh, D., 2000. Behaviour Studying Models in Health Education. 1st Edn. Tehran. Health education and Communication Administration Publication, pp: 20-40.

8. Francis, K., 1997. Health BehAviour and Health Education. 1st Edn. Tehran, Ladan Co., pp: 64-71.

9. Ryan, M.A. and R.S. Christian, 2001. Hand washing and respiratory illness among young adults in military training. Am. J. Prev. Med.,, 21: 150-8.

10. McElroy, B.H. and S.P. Miller, 2003. Effectiveness of zinc gluconate lozenges. Am. J. Ther., 10: 233.

11. Jackson, G. and H. Dowling, 1958. Transmission of the common cold to the volunteers under controlled conditions. Arc. Int. Med, 101: 267-78.

12. Sattar, S.A. and H. Jacobsen 1993. Chemical disinfection to interrupt transfer of rhinovirus type 14 from environmental surfaces to hand. Appl. Environ. Microbiol., 59: 1579-85.

13. Niffeneger, J.P., 1997. Proper hand washing promotes wellness in child care. J. Pediatr. Health Care, 11: 26-31.

14. Belongia, E.A. and R. Berg, 2001. A randomized trial of zinc nasal spray for the treatment of upper respiratory illness in adults. Am. J. Med., 111: 103108. 